\title{
Impact of lighting color and duration on productive performance and Newcastle disease vaccination efficiency in broiler chickens
}

\author{
Essam S. Soliman ${ }^{1}$ and Rania A. Hassan²
}

1. Department of Animal Hygiene, Zoonosis, and Animal Behavior, Faculty of Veterinary Medicine, Suez Canal University, Ismailia 41522, Egypt; 2. Department of Animal Wealth Development, Animal Production Division, Faculty of Veterinary Medicine, Suez Canal University, Ismailia 41522, Egypt.

Corresponding author: Essam S. Soliman, e-mail: soliman.essam@vet.suez.edu.eg Co-author: RAH: rania_tamer_178@yahoo.com

Received: 05-03-2019, Accepted: 10-06-2019, Published online: 17-07-2019

doi: 10.14202/vetworld.2019.1052-1059 How to cite this article: Soliman ES, Hassan RA (2019) Impact of lighting color and duration on productive performance and Newcastle disease vaccination efficiency in broiler chickens, Veterinary World, 12(7): 1052-1059.

\begin{abstract}
Background and Aim: Manipulating lighting colors and regimens is considered an effective mean for improving broiler productivity. The influence of red, blue, and white light-emitting diode (LED) was investigated using three different regimens of lighting and darkness; continuous $23 \mathrm{~h}$ light (L): $1 \mathrm{~h}$ dark (D), continuous $18 \mathrm{~h} \mathrm{~L}: 6 \mathrm{~h} \mathrm{D}$, and intermittent $16 \mathrm{~h}$ $\mathrm{L}: 8 \mathrm{~h}$ D hours on the performance, carcass weight (CW), feed and water intake (WI), serum glucose (GLUCO), triglycerides (TG), and cholesterol (TC), intestinal bacterial load, growth and metabolic hormones, and efficiency of Newcastle disease (ND) vaccine.

Materials and Methods: A total of 2521 -day-old Ross broilers on deep litter were divided into nine groups. The $1^{\text {st }}, 4^{\text {th }}$, and $7^{\text {th }}$ groups were exposed to continuous $23 \mathrm{~L}: 1 \mathrm{D}$, the $2^{\text {nd }}, 5^{\text {th }}$, and $8^{\text {th }}$ groups were exposed to continuous $18 \mathrm{~L}: 6 \mathrm{D}$, and the $3^{\text {rd }}$, $6^{\text {th }}$, and $9^{\text {th }}$ groups were exposed to intermittent $16 \mathrm{~L}: 8 \mathrm{D}(4 \mathrm{~L}: 2 \mathrm{D}, 4$ times) lighting regimen using red, blue, and white LED lights, respectively. A total of 1350 samples (225 sera, 225 swabs, and 900 organ samples) were collected.

Results: Blue LED group revealed a highly significant increase $(p<0.01)$ in live body weight, body weight gain, performance index, CW, spleen, heart, and liver weights, and anti-ND antibody titer, as well as a highly significant decline $(\mathrm{p}<0.01)$ of feed intake, WI, GLUCO, TG, TC, growth hormone, insulin, tri-iodothyronine (T3), tetra-iodothyronine (T4), total bacterial count (TBC), and total Enterobacteriaceae count compared to red and white LED lights in all tested lighting regimens. Continuous 23L:1D and 18L:6D regimens were significantly $(p<0.01)$ superior to intermittent 16L:8D in their influence on the performance, $\mathrm{CW}$, biochemistry, hormonal profile, and bacterial load.
\end{abstract}

Conclusion: The blue LED light associated with continuous 18L:6D or 23L:1D h regimen is highly recommended in broiler houses for their enhancing the productive performance, growth, and immunity.

Keywords: broilers, light-emitting diode colors, metabolism, Newcastle vaccine, productive performance.

\section{Introduction}

Light is a key microclimatic factor that hits broiler skull at the retinal receptors and traveling through neurons to the pineal gland, stimulating pineal gland, and hypothalamus regulating functions including metabolism and reproduction [1]. Light is known for its influence on growth performance, immunity levels, metabolism, behavior, and bird activity [2,3]. The eye and visual cortex represent anatomically a large proportion in the broiler brain. Eyes are important in realizing the extension of the landscape, recognizing features of other birds, and providing a probable response to humans. Broilers perceive light signals from the surrounding microclimate through photoreceptors that consist of one rod and six cones.

Copyright: Soliman and Hassan. Open Access. This article is distributed under the terms of the Creative Commons Attribution 4.0 International License (http://creativecommons.org/licenses/ by/4.0/), which permits unrestricted use, distribution, and reproduction in any medium, provided you give appropriate credit to the original author(s) and the source, provide a link to the Creative Commons license, and indicate if changes were made. The Creative Commons Public Domain Dedication waiver (http:// creativecommons.org/publicdomain/zero/1.0/) applies to the data made available in this article, unless otherwise stated.
Photosensitive pigments in retinal rods and cones relay these light signals to central neurons, where signals are integrated into an image [4].

Light color (wavelength) is an important component of the physical light environment that affects broiler growth, performance, and welfare. Long wavelengths are known for higher penetration power compared to short wavelengths [5]. Artificial lighting has been used in modern poultry production to stimulate productive performance. The light-emitting diode (LED) provides an approximation of daylight than the spectral gaps of other lighting sources [6]. Researches conducted on light color suggested the blue, green lights [7,8], and white lights [9] to enhance broiler growth, production, and act on increasing myofiber growth through effective stimulation of testosterone.

Photoperiod is defined as the length of light duration, while scotoperiod is the length of dark hours. Lighting regimens in the poultry industry have been fluctuated between continuous and intermittent lighting according to their effectiveness on welfare and productive performance of broilers $[10,11]$. Continuous lighting systems combined with ad libitum feeding 
adversely affected broilers performance compared to restricted lighting' and feeding regimen [12]. Modern poultry production was associated with intensification and genetic selection for rapidly growing broilers, that is, why many researchers investigated and noted that a longer photoperiod and a shortened scotoperiod contributed to increased livability and feed intake (FI), and thus increased weight gain (WG) in broilers [13].

This study aimed to investigate the influence of manipulating lighting color as red, blue, and white LED and duration (continuous 23L:1D, continuous 18L:6D, and intermittent 16L:8D hours) on productive performance, feed and water intake (WI), carcass weight $(\mathrm{CW})$, immune organs' (spleen and bursa) and edible organs' (liver and heart) weights, biochemical parameters (glucose [GLUCO], triglycerides [TG], and total cholesterol [TC]), intestinal bacterial load, growth and metabolic hormones, and efficiency of Newcastle virus vaccine.

\section{Materials and Methods}

Ethical approval

The protocol of the present study was approved by the Scientific Research Ethics Committee of Faculty of Veterinary Medicine, Suez Canal University, Ismailia, Egypt, (2018061).

\section{Experimental plan and housing management}

A total of 252 1-day-old Ross broilers were purchased from El-Frog Co-Ismailia, divided into nine groups of 28 broilers each (four replicates, each one seven chicks), and housed on a deep litter system. The floor of the building was treated with superphosphate $0.5 \mathrm{~g} / \mathrm{m}^{2}$, according to Soliman et al. [14] to absorb moisture, minimize ammonia evaporation, and reduce microbial survival.

The building was divided interiorly using dark brown blackout curtains into nine sections, each of $3 \mathrm{~m}^{2}$ and used for one group and its assigned lighting program. Each section was provided with a V-shaped window that was covered with dark blackout to prevent their interference with the lighting program and a ceiling fan to encourage air exchange and stimulate stack effect. An automated LED lamp of 18 watt, 1750 lumen, and monochromic (red, blue, and white) was supplied in each section and adjusted by timer according to the recommended light (L)/dark (D) hours for each group. The experiment was designed to past for 40 days, during which mortalities, temperature, and relative humidity were monitored daily.

\section{Broiler microclimate}

Broilers were brooded at $35^{\circ} \mathrm{C}$ and decreased by $3^{\circ} \mathrm{C}$ weekly until achieving $21-24^{\circ} \mathrm{C}$ by the $3^{\text {rd }}$ week. Broilers were supplied with standard corn-soybean ration, as shown in Table-1, to satisfy the basic requirements as recommended by the National Research Council [15]. In addition, they were given ad libitum access to water. Broilers were vaccinated using drinking water mass vaccination with live attenuated
Table-1: Feed ingredients and nutrient contents of standard soybean ration at different growth stages of the experiment.

\begin{tabular}{lcc}
\hline Ingredients \% & $\begin{array}{c}\text { Starter } \\
\mathbf{1 : 1 4} \text { days }\end{array}$ & $\begin{array}{c}\text { Grower } \\
\mathbf{1 5 : 4 0} \text { days }\end{array}$ \\
\hline Corn & 50.00 & 56.50 \\
Soybean meal & 39.00 & 33.50 \\
Fish meal & 3.00 & 2.50 \\
Calcium carbonate & 2.00 & 2.00 \\
Monocalcium phosphate & 2.00 & 2.00 \\
DL-methionine & 0.50 & 0.50 \\
L-lysine & 0.50 & 0.50 \\
Vitamin mix & 2.50 & 2.50 \\
Common salt & 0.50 & 0.50 \\
Energy & $2990 \mathrm{Kcal} / \mathrm{kg}$ & $3200 \mathrm{Kcal} / \mathrm{kg}$ \\
Protein & $22 \%$ & $21 \%$ \\
Fat & $3.5 \%$ & $5.5 \%$ \\
Crude fiber & $3.4 \%$ & $3.7 \%$ \\
\hline
\end{tabular}

virus of IB-H120 $\geq 10^{3.5}$ against infectious bronchitis at day 6, initial and booster doses of live attenuated virus of VMG91 $\geq 10^{3.0}$ against infectious bursal disease at days 14 and 21, respectively, and with initial and booster doses of live lentogenic Newcastle disease (ND) virus of Lasota $\geq 10^{6.0}$ against (ND) virus at days 18 and 28, respectively.

\section{Lighting colors and durations regimens}

Broilers of Group 1 (G1), Group 2 (G2), and Group 3 (G3) were exposed to red LED light, Group 4 (G4), Group 5 (G5), and Group 6 (G6) were exposed to blue LED light, and Group 7 (G7), Group 8 (G8), and Group 9 (G9) were exposed to white LED light. Light (L) to dark (D) hours were adjusted automatically by timer in each group as following: G1, G4, and G7 were exposed to continuous lighting using 23L:1D hours regimen, G2, G5, and G8 were exposed to continuous lighting using 18L:6D hours regimen, and G3, G6, and G9 were exposed to intermittent lighting using 16L:8D hours regimen (4L: 2D, 4 times).

\section{Performance indices (PI)}

$\mathrm{FI} / \mathrm{g}$ of each bird was calculated from the total amount consumed based on each group's intensity (the absolute number of birds in each group). Live body weight (LBW/g) was estimated by weighing at least 26 birds per group every week, the number was calculated by simple random sampling design as recommended by Thrusfield [16] with an expected error 5\%:

$$
\mathrm{n}=1.96^{2} \mathrm{P}_{\text {exp }}\left(1-\mathrm{P}_{\text {exp }}\right) / \mathrm{d}^{2}
$$

Where $\mathrm{n}=$ required sample size, $\mathrm{P}_{\text {exp }}=$ Expected prevalence, $\mathrm{d}=$ Desired absolute precision. Performance indices including body WG (BWG/g), feed conversion ratio (FCR), and performance index (PI) were calculated as recommended by Soliman and Hassan [17]. Mortalities were calculated as a proportion between the numbers of succumbed birds from the total population at risk.

\section{Sampling}

A total of 1350 samples (225 sera, 225 intestinal swabs, and 900 organ samples including edible organs 
as liver and heart, and immune organs as spleen and bursa) were collected by the end of the experiment (40 days). Blood samples were collected, held at $37^{\circ} \mathrm{C}$ for $2 \mathrm{~h}$, and centrifuged at $3500 \mathrm{rpm}$ for $15 \mathrm{~min}$. Clear sera were dispensed into Eppendorf tubes, tested for blood sugars, and stored at $-20^{\circ} \mathrm{C}$ for biochemical, hormonal, and immunological analysis [18]. Birds were slaughtered after blood sampling; carcasses were weighed (total $\mathrm{CW}$ and expressed by $\mathrm{CW} / \mathrm{g}$ ), liver, heart, spleen, and bursa were removed, weighed, and expressed as $\mathrm{g} / \mathrm{kg}$. Swab samples were collected from the intestine, added to $9 \mathrm{~mL}$ buffered peptone water, preserved in an icebox and transferred to the laboratory for bacteriological evaluation.

\section{Biochemical and hormonal profile}

Sera were examined for GLUCO $\mathrm{mg} / \mathrm{dl}$, TG mg/dl, and TC mg/dl calorimetrically using Roche COBAS INTEGRA ${ }^{\circledR} 400$ Plus Analyzer. Growth hormone $(\mathrm{GH} \mathrm{ng} / \mathrm{mL})$, insulin $(\mu \mathrm{IU} / \mathrm{mL})$, tri-iodothyronine $(\mathrm{T} 3 \mathrm{ng} / \mathrm{mL})$, tetra-iodothyronine $(\mathrm{T} 4 \mathrm{ng} / \mathrm{mL})$, and Newcastle virus vaccine titer (ND $\mathrm{mg} / \mathrm{dl}$ ) were measured using Roche ELECSYS 1010 Immunoassay Analyzer [19].

\section{Bacteriological examination}

Intestinal swabs were subjected to ten-fold serial dilutions up to $10^{-6}$, as recommended by APHA [20]. Total bacterial count (TBC) onto standard plate count agar and total Enterobacteriaceae count (TEC) onto eosin methylene blue agar at $37^{\circ} \mathrm{C}$ for $24-48 \mathrm{~h}$ were performed using a drop plate technique [21,22]. Plates were counted using Darkfield Colony Counter [23].

\section{Statistical analysis}

Statistical analysis was carried out using a Statistical Package for the Social Sciences version 20 (IBM SPSS Statistics 20) [24]. The obtained data were analyzed statistically using a multifactorial analysis of variance. Bacterial counts were transferred into logarithmic counts using Microsoft Excel.

\section{Results}

Crude mortality rates revealed a total of $8.7 \%$ (22 out of 252 birds), including $35.71 \%$ (10 out of 28 birds), $17.85 \%$ (5 out of 28 birds), $10.71 \%$ ( 3 out of 28 birds), and $14.28 \%$ (4 out of 28 birds) mortality rates in broilers raised in continuous 23L:1D hours regimen of red, intermittent 16L:8D hours regimen of red, intermittent 16L:8D hours regimen of blue, and intermittent 16L:8D hours regimen of white light system, respectively.

Performance indices, in Table-2, revealed a highly significant increase $(\mathrm{p}<0.01)$ of $\mathrm{BWG}$ and PI among broilers reared in continuous 23L:1D and 18L:6D hours regimens of blue light compared to intermittent lighting regimen. Meanwhile, the lowest significant $(\mathrm{p}<0.01)$ FCR was recorded in continuous 23L:1D hours regimen of blue light.

WI revealed a highly significant increase $(p<0.01)$, as shown in Table-2, between broilers raised in continuous 23L:1D hours regimen and those raised in intermittent 16L:8D hours of red light with no significant differences between neither of them and continuous 18L:6D. FI revealed no significant difference in the overall comparison between all broiler groups raised under different systems. On calculating WI/FI ratio (Table-2), no significant differences were revealed between the nine lighting regimens.

Continuous 23L:1D and 18L:6D hours regimens of blue light revealed a highly significant increase $(p<0.01)$ of LBW, CW, spleen, and heart weights

Table-2: Performance indices (Mean \pm SE) in broilers exposed to lighting regimens using different lighting colors and durations.

\begin{tabular}{|c|c|c|c|c|c|c|c|}
\hline Light color & Photo-period L: D hour & BWG/g & FCR \% & PI & $\mathbf{W I} / \mathbf{m L}$ & $\mathbf{F I} / \mathbf{g}$ & WI: FI ratio \\
\hline$\overline{R e d}$ & & $253.0^{c} \pm 13.0$ & $2.18^{\mathrm{a}} \pm 0.07$ & $3.3^{c} \pm 0.21$ & $530.8^{\mathrm{a}} \pm 28.1$ & $206.2^{\mathrm{a}} \pm 16.4$ & $0.35^{\mathrm{a}} \pm 0.012$ \\
\hline Blue & & $391.8^{a} \pm 21.7$ & $1.14^{c} \pm 0.04$ & $9.4^{\mathrm{a}} \pm 0.78$ & $433.9^{b} \pm 23.1$ & $163.8^{\mathrm{a}} \pm 13.0$ & $0.34^{\mathrm{a}} \pm 0.013$ \\
\hline White & & $329.5^{b} \pm 15.5$ & $1.43^{\mathrm{b}} \pm 0.06$ & $6.3^{b} \pm 0.40$ & $466.5^{\mathrm{ab}} \pm 24.6$ & $190.7^{a} \pm 14.8$ & $0.37^{\mathrm{a}} \pm 0.013$ \\
\hline $\mathrm{p}$-value & & 0.000 & 0.002 & 0.000 & 0.026 & 0.056 & 0.310 \\
\hline \multicolumn{8}{|c|}{ Light's color $X$ lighting program } \\
\hline \multicolumn{8}{|l|}{ Red } \\
\hline & $C-23: 1$ & $270.7^{a} \pm 24.7$ & $2.16^{\mathrm{b}} \pm 0.14$ & $3.6^{\mathrm{a}} \pm 0.43$ & $556.3^{a} \pm 50.1$ & $224.9^{a} \pm 30.5$ & $0.37^{\mathrm{a}} \pm 0.021$ \\
\hline & $C-18: 6$ & $258.5^{b} \pm 22.4$ & $2.10^{c} \pm 0.11$ & $3.4^{b} \pm 0.37$ & $530.2^{\mathrm{ab}} \pm 49.6$ & $205.3^{b} \pm 28.6$ & $0.35^{\mathrm{a}} \pm 0.020$ \\
\hline & $\mathrm{I}-16: 8$ & $229.8^{c} \pm 20.6$ & $2.30^{\mathrm{a}} \pm 0.13$ & $2.9^{c} \pm 0.29$ & $505.9^{b} \pm 47.6$ & $188.2^{\mathrm{c}} \pm 26.9$ & $0.33^{\mathrm{a}} \pm 0.021$ \\
\hline \multicolumn{8}{|c|}{ 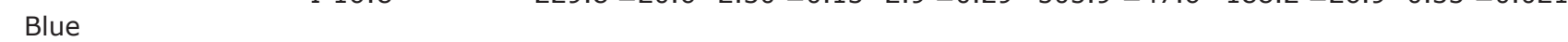 } \\
\hline & $C-23: 1$ & $415.6^{a} \pm 38.9$ & $1.12^{\mathrm{c}} \pm 0.07$ & $9.6^{\mathrm{a}} \pm 1.31$ & $464.1^{\mathrm{a}} \pm 44.2$ & $170.1^{\mathrm{a}} \pm 23.3$ & $0.33^{a} \pm 0.018$ \\
\hline & $C-18: 6$ & $384.4^{b} \pm 37.1$ & $1.15^{\mathrm{b}} \pm 0.08$ & $9.3^{b} \pm 1.42$ & $425.0^{\mathrm{ab}} \pm 38.1$ & $163.0^{\mathrm{b}} \pm 22.7$ & $0.35^{a} \pm 0.025$ \\
\hline & I-16:8 & $375.4^{c} \pm 38.0$ & $1.16^{\mathrm{a}} \pm 0.09$ & $9.1^{\mathrm{c}} \pm 1.40$ & $412.6^{b} \pm 38.2$ & $158.5^{\complement} \pm 22.6$ & $0.34^{\mathrm{a}} \pm 0.025$ \\
\hline \multicolumn{8}{|c|}{ 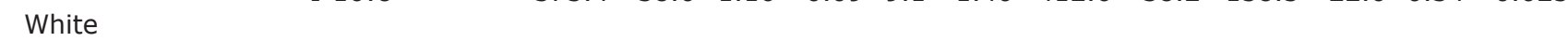 } \\
\hline & $C-23: 1$ & $352.7^{a} \pm 26.4$ & $1.39^{c} \pm 0.10$ & $6.8^{\mathrm{a}} \pm 0.67$ & $497.1^{a} \pm 46.3$ & $201.5^{\mathrm{a}} \pm 26.5$ & $0.37^{\mathrm{a}} \pm 0.019$ \\
\hline & $C-18: 6$ & $325.0^{b} \pm 28.2$ & $1.45^{\mathrm{a}} \pm 0.11$ & $6.1^{b} \pm 0.74$ & $462.8^{\mathrm{ab}} \pm 42.2$ & $189.4^{b} \pm 26.2$ & $0.37^{a} \pm 0.023$ \\
\hline & I-16:8 & $310.7^{c} \pm 26.2$ & $1.44^{b} \pm 0.12$ & $5.9^{c} \pm 0.68$ & $439.5^{b} \pm 40.3$ & $181.3^{c} \pm 25.3$ & $0.37^{a} \pm 0.024$ \\
\hline p-value & & 0.009 & 0.003 & 0.007 & 0.000 & 0.024 & 0.860 \\
\hline
\end{tabular}

Means carrying different superscripts in the same column are significantly different at $(p \leq 0.05)$ or highly significantly different at $p<0.01$. Means carrying the same superscripts in the same column are non-significantly different at $p<0.05$. $\mathrm{L}=$ Light hours, $\mathrm{D}=$ Dark hours, $\mathrm{C}=$ Continuous, $\mathrm{I}=$ Intermittent, $\mathrm{BWG}=$ Body weight gain, $\mathrm{FCR}=$ Feed conversion ratio, $\mathrm{PI}=$ Performance index, WI=Water intake, FI=Feed intake, WI: FI=Water intake to Feed intake ratio, SE=Standard error 
compared to other lighting colors and regimens (Table-3). Furthermore, liver and bursa revealed a highly significant increase $(p<0.01)$ in broilers raised in continuous $23 \mathrm{~L}: 1 \mathrm{D}$ and $18 \mathrm{~L}: 6 \mathrm{D}$ hours regimens of blue and red lights with no significant differences in between (Table-3).

GLUCO, TG, and TC revealed, in Table- 4 , the highest significance $(p<0.01)$ in red light. Meanwhile, the lowest significance $(p<0.01)$ of TG and TC was recorded in blue and white lights. A synchronized highly significant decline $(p<0.01)$ of GLUCO, TG, and TC was recorded, as shown in Table- 4 , in continuous $23 \mathrm{~L}: 1 \mathrm{D}$, continuous $18 \mathrm{~L}: 6 \mathrm{D}$, and intermittent 16L:8D hours regimens, respectively.

$\mathrm{GH}$, insulin, T3, and T4 hormones, in Table-5, revealed a highly significant increase $(\mathrm{p}<0.01)$ in broilers reared in red light compared to those reared in blue and white lights. Meanwhile, ND titer revealed a highly significant increase $(p<0.01)$ in blue, white, and red lights, respectively. GH and ND vaccine titer

Table-3: Terminal marketing body weight and carcass quality characteristics (Mean $\pm \mathrm{SE}$ ) in broilers exposed to lighting regimens using different lighting colors and durations.

\begin{tabular}{|c|c|c|c|c|c|c|c|}
\hline \multirow[t]{2}{*}{ Light color } & \multirow[t]{2}{*}{ Photo-period L: D hour } & \multirow[t]{2}{*}{ LBW/g } & \multirow[t]{2}{*}{$\mathbf{C W} / \mathrm{g}$} & \multicolumn{4}{|c|}{ Organs/CW ratio } \\
\hline & & & & Liver \% & Spleen \% & Heart \% & Bursa \% \\
\hline $\begin{array}{l}\text { Red } \\
\text { Blue } \\
\text { White } \\
\text { p-value }\end{array}$ & & $\begin{array}{c}1300^{\mathrm{c}} \pm 12.5 \\
2028^{\mathrm{a}} \pm 10.9 \\
1703^{\mathrm{b}} \pm 12.7 \\
0.002\end{array}$ & $\begin{array}{c}1007^{\mathrm{c}} \pm 11.6 \\
1749^{\mathrm{a}} \pm 9.1 \\
1418^{\mathrm{b}} \pm 10.3 \\
0.000\end{array}$ & $\begin{array}{c}1.90^{\mathrm{a}} \pm 0.04 \\
1.98^{\mathrm{a}} \pm 0.04 \\
1.68^{\mathrm{b}} \pm 0.04 \\
0.000\end{array}$ & $\begin{array}{c}0.05^{\mathrm{c}} \pm 0.00 \\
0.09^{\mathrm{a}} \pm 0.00 \\
0.08^{\mathrm{b}} \pm 0.01 \\
0.000\end{array}$ & $\begin{array}{c}0.34^{\mathrm{c}} \pm 0.01 \\
0.47^{\mathrm{a}} \pm 0.02 \\
0.40^{\mathrm{b}} \pm 0.02 \\
0.000\end{array}$ & $\begin{array}{c}0.12^{\mathrm{a}} \pm 0.00 \\
0.06^{\mathrm{b}} \pm 0.00 \\
0.05^{\mathrm{b}} \pm 0.00 \\
0.096\end{array}$ \\
\hline \multicolumn{8}{|c|}{ Light's color $X$ lighting program } \\
\hline \multicolumn{8}{|l|}{ Red } \\
\hline & $C-23: 1$ & $1382^{\mathrm{a}} \pm 17.3$ & $1086^{\mathrm{a}} \pm 11.4$ & $2.0^{\mathrm{a}} \pm 0.07$ & $0.06^{a} \pm 0.00$ & $0.39^{a} \pm 0.01$ & $0.13^{\mathrm{a}} \pm 0.01$ \\
\hline & $\begin{array}{l}\text { C-18:6 } \\
\mathrm{I}-16: 8\end{array}$ & $\begin{array}{l}1348^{\mathrm{b}} \pm 5.3 \\
1171^{\mathrm{c}} \pm 7.7\end{array}$ & $\begin{array}{c}1059^{\mathrm{a}} \pm 7.2 \\
876^{\mathrm{b}} \pm 4.9\end{array}$ & $\begin{array}{l}1.7^{c} \pm 0.06 \\
1.9^{b} \pm 0.08\end{array}$ & $\begin{array}{l}0.05^{b} \pm 0.00 \\
0.05^{b} \pm 0.00\end{array}$ & $\begin{array}{l}0.31^{b} \pm 0.01 \\
0.30^{b} \pm 0.02\end{array}$ & $\begin{array}{l}0.12^{b} \pm 0.01 \\
0.11^{c} \pm 0.01\end{array}$ \\
\hline \multicolumn{8}{|l|}{ Blue } \\
\hline & $C-23: 1$ & $2150^{\mathrm{a}} \pm 9.7$ & $1896^{\mathrm{a}} \pm 10.2$ & $2.2^{\mathrm{a}} \pm 0.05$ & $0.11^{\mathrm{a}} \pm 0.01$ & $0.57^{a} \pm 0.02$ & $0.07^{\mathrm{a}} \pm 0.00$ \\
\hline & $C-18: 6$ & $1990^{b} \pm 5.3$ & $1712^{\mathrm{b}} \pm 6.2$ & $2.0^{\mathrm{b}} \pm 0.05$ & $0.09^{b} \pm 0.01$ & $0.47^{b} \pm 0.03$ & $0.06^{a} \pm 0.01$ \\
\hline & $\mathrm{I}-16: 8$ & $1944^{c} \pm 3.8$ & $1638^{c} \pm 4.1$ & $1.6^{c} \pm 0.05$ & $0.08^{c} \pm 0.01$ & $0.37^{c} \pm 0.03$ & $0.05^{\mathrm{a}} \pm 0.01$ \\
\hline \multicolumn{8}{|c|}{ 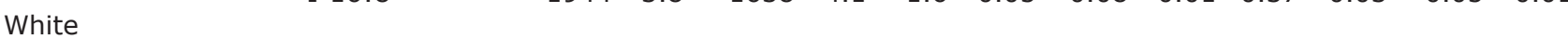 } \\
\hline & $C-23: 1$ & $1847^{a} \pm 10.9$ & $1568^{\mathrm{a}} \pm 9.8$ & $1.8^{\mathrm{a}} \pm 0.06$ & $0.08^{\mathrm{a}} \pm 0.01$ & $0.45^{\mathrm{a}} \pm 0.03$ & $0.06^{a} \pm 0.01$ \\
\hline & $C-18: 6$ & $1657^{b} \pm 5.1$ & $1376^{\mathrm{b}} \pm 4.9$ & $1.7^{\mathrm{b}} \pm 0.07$ & $0.07^{b} \pm 0.01$ & $0.39^{b} \pm 0.03$ & $0.05^{\mathrm{a}} \pm 0.01$ \\
\hline & $\mathrm{I}-16: 8$ & $1606^{c} \pm 3.6$ & $1310^{\mathrm{b}} \pm 3.2$ & $1.5^{\complement} \pm 0.07$ & $0.08^{\mathrm{a}} \pm 0.01$ & $0.35^{c} \pm 0.04$ & $0.04^{a} \pm 0.01$ \\
\hline p-value & & 0.000 & 0.000 & 0.002 & 0.068 & 0.005 & 0.006 \\
\hline
\end{tabular}

Means carrying different superscripts in the same column are significantly different at $p \leq 0.05$ or highly significantly different at $p<0.01$. Means carrying the same superscripts in the same column are non-significantly different at $p<0.05$. $L=$ Light hours, $\mathrm{D}=$ Dark hours, $\mathrm{C}=$ Continuous, $\mathrm{I}=$ Intermittent, $\mathrm{LBW}=$ Live body weight, $\mathrm{CW}=$ Carcass weight, $\mathrm{SE}=\mathrm{Standard}$ error

Table-4: Biochemical profile (Mean \pm SE) in broilers exposed to lighting regimens using different lighting colors and durations.

\begin{tabular}{|c|c|c|c|c|}
\hline Light color & Photo-period L: D hour & GLUCO mg/dl & TG mg/dl & TC mg/dl \\
\hline Red & & $237.1^{\mathrm{a}} \pm 5.03$ & $235.7^{\mathrm{a}} \pm 2.42$ & $317.1^{\mathrm{a}} \pm 6.92$ \\
\hline Blue & & $157.8^{\mathrm{b}} \pm 4.84$ & $108.3^{c} \pm 1.90$ & $148.7^{c} \pm 4.56$ \\
\hline White & & $118.0^{c} \pm 3.87$ & $144.0^{\mathrm{b}} \pm 2.24$ & $247.7^{b} \pm 5.55$ \\
\hline p-value & & 0.000 & 0.000 & 0.000 \\
\hline \multicolumn{5}{|c|}{ Light's color $X$ lighting program } \\
\hline \multicolumn{5}{|l|}{ Red } \\
\hline & $C-23: 1$ & $184.2^{\mathrm{c}} \pm 2.12$ & $218.4^{\mathrm{c}} \pm 2.51$ & $246.8^{c} \pm 2.43$ \\
\hline & $C-18: 6$ & $240.1^{b} \pm 2.16$ & $230.2^{b} \pm 1.56$ & $314.7^{b} \pm 3.11$ \\
\hline & I-16:8 & $287.2^{\mathrm{a}} \pm 2.03$ & $258.4^{\mathrm{a}} \pm 1.09$ & $389.7^{a} \pm 2.55$ \\
\hline \multicolumn{5}{|l|}{ Blue } \\
\hline & $C-23: 1$ & $114.7^{c} \pm 2.15$ & $88.2^{\complement} \pm 0.91$ & $108.3^{c} \pm 2.71$ \\
\hline & $C-18: 6$ & $147.1^{\mathrm{b}} \pm 2.18$ & $110.1^{b} \pm 0.56$ & $140.4^{b} \pm 2.11$ \\
\hline & $\mathrm{I}-16: 8$ & $211.7^{\mathrm{a}} \pm 1.55$ & $126.6^{\mathrm{a}} \pm 0.87$ & $197.5^{\mathrm{a}} \pm 1.87$ \\
\hline \multicolumn{5}{|l|}{ White } \\
\hline & $C-23: 1$ & $79.7^{c} \pm 1.49$ & $121.8^{\mathrm{c}} \pm 1.69$ & $184.7^{c} \pm 1.19$ \\
\hline & $C-18: 6$ & $115.0^{\mathrm{b}} \pm 1.08$ & $146.1^{b} \pm 1.52$ & $259.6^{b} \pm 1.11$ \\
\hline & I-16: 8 & $159.1^{\mathrm{a}} \pm 1.14$ & $164.2^{\mathrm{a}} \pm 1.22$ & $299.0^{a} \pm 0.98$ \\
\hline p-value & & 0.000 & 0.002 & 0.000 \\
\hline
\end{tabular}

Means carrying different superscripts in the same column are significantly different at $\mathrm{p} \leq 0.05$ or highly significantly different at $p<0.01$. Means carrying the same superscripts in the same column are non-significantly different at $p<0.05$. $\mathrm{L}=$ Light hours, $\mathrm{D}=$ Dark hours, $\mathrm{C}=$ Continuous, $\mathrm{I}=$ Intermittent, $\mathrm{GLUCO}=\mathrm{Glucose}, \mathrm{TG}=$ Triglycerides, $\mathrm{TC}=$ Total cholesterol, $\mathrm{SE}=$ Standard error 
revealed a highly significant increase $(\mathrm{p}<0.01)$ in continuous 23L:1D, continuous $18 \mathrm{~L}: 6 \mathrm{D}$, and intermittent 16L:8D hours regimens, respectively, although that insulin, T3, and T4 hormones revealed a highly significant decline $(p<0.01)$ in the same lighting regimens (Table-5).

Log TBC and TEC showed, in Table-6, a highly significant decline $(p<0.01)$ in continuous $23 \mathrm{~L}: 1 \mathrm{D}$, continuous 18L:6D, and intermittent 16L:8D hours regimens of blue, white, and red lighting regimens, respectively.

\section{Discussion}

Modern broiler industry has been focusing on genetically selected broilers for fast growth and rapid WG at the expense of livability, immunity, and leg problems that may be developed. Challenging conditions demand control over the early growth in broilers through conserving feed conversion and livability with a good opportunity for lungs, heart, and skeletal muscles to develop before muscle tissue rapid formation [25]. Light programs have been manipulated in the broiler industry to capture high gain during

Table-5: Hormonal profile and Newcastle virus vaccine titer (Mean \pm SE) in broilers exposed to lighting regimens using different lighting colors and durations.

\begin{tabular}{|c|c|c|c|c|c|c|}
\hline Light color & Photo-period L: D hour & GH $\mathbf{n g} / \mathbf{m L}$ & Insulin $\mu \mathrm{IU} / \mathbf{m L}$ & T3 ng/mL & T4 ng/mL & ND Titer $\mathbf{m g} / \mathbf{d l}$ \\
\hline Red & & $109.8^{\mathrm{a}} \pm 0.78$ & $20.5^{a} \pm 0.16$ & $202.2^{\mathrm{a}} \pm 1.46$ & $12.2^{\mathrm{a}} \pm 0.11$ & $212.4^{c} \pm 8.15$ \\
\hline Blue & & $19.4^{b} \pm 1.76$ & $15.3^{\mathrm{b}} \pm 0.30$ & $107.5^{\complement} \pm 3.04$ & $5.7^{c} \pm 0.21$ & $420.7^{a} \pm 8.10$ \\
\hline White & & $18.5^{\complement} \pm 0.23$ & $7.2^{c} \pm 0.18$ & $150.7^{b} \pm 2.32$ & $7.5^{b} \pm 0.19$ & $307.1^{\mathrm{b}} \pm 4.35$ \\
\hline p-value & & 0.000 & 0.000 & 0.002 & 0.005 & 0.001 \\
\hline \multicolumn{7}{|c|}{ Light's color $X$ lighting program } \\
\hline \multicolumn{7}{|l|}{ Red } \\
\hline & $C-23: 1$ & $117.5^{\mathrm{a}} \pm 0.72$ & $18.7^{c} \pm 0.09$ & $189.1^{\complement} \pm 1.25$ & $11.3^{c} \pm 0.13$ & $286.1^{\mathrm{a}} \pm 3.93$ \\
\hline & $C-18: 6$ & $108.5^{b} \pm 0.68$ & $20.8^{\mathrm{b}} \pm 0.08$ & $201.1^{\mathrm{b}} \pm 1.33$ & $12.2^{\mathrm{b}} \pm 0.09$ & $228.7^{b} \pm 3.36$ \\
\hline & $\mathrm{I}-16: 8$ & $103.5^{\complement} \pm 0.55$ & $22.0^{a} \pm 0.09$ & $216.2^{\mathrm{a}} \pm 1.19$ & $13.0^{\mathrm{a}} \pm 0.11$ & $123.2^{\mathrm{c}} \pm 2.98$ \\
\hline \multicolumn{7}{|l|}{ Blue } \\
\hline & $C-23: 1$ & $40.7^{a} \pm 0.66$ & $12.0^{c} \pm 0.12$ & $77.6^{c} \pm 0.74$ & $3.6^{c} \pm 0.07$ & $497.7^{a} \pm 4.04$ \\
\hline & $C-18: 6$ & $9.71^{b} \pm 0.11$ & $15.8^{\mathrm{b}} \pm 0.11$ & $103.9^{b} \pm 0.82$ & $5.5^{b} \pm 0.08$ & $429.5^{b} \pm 3.56$ \\
\hline & I-16: 8 & $8.0^{\wedge} \pm 0.12$ & $18.1^{\mathrm{a}} \pm 0.09$ & $140.9^{\mathrm{a}} \pm 0.77$ & $8.1^{\mathrm{a}} \pm 0.07$ & $334.6^{c} \pm 4.12$ \\
\hline \multicolumn{7}{|c|}{ 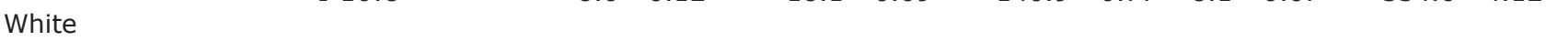 } \\
\hline & $C-23: 1$ & $20.7^{a} \pm 0.17$ & $5.4^{\complement} \pm 0.08$ & $126.0^{\complement} \pm 1.32$ & $5.6^{c} \pm 0.09$ & $352.1^{\mathrm{a}} \pm 2.94$ \\
\hline & $C-18: 6$ & $18.6^{b} \pm 0.18$ & $7.2^{b} \pm 0.07$ & $154.0^{\mathrm{b}} \pm 0.99$ & $7.4^{b} \pm 0.08$ & $301.3^{\mathrm{b}} \pm 2.22$ \\
\hline & I-16:8 & $16.3^{c} \pm 0.23$ & $9.6^{a} \pm 0.08$ & $172.1^{\mathrm{a}} \pm 1.41$ & $9.5^{a} \pm 0.08$ & $268.4^{c} \pm 2.54$ \\
\hline p-value & & 0.000 & 0.000 & 0.000 & 0.002 & 0.003 \\
\hline
\end{tabular}

Means carrying different superscripts in the same column are significantly different at $p \leq 0.05$ or highly significantly different at $p<0.01$. Means carrying the same superscripts in the same column are non-significantly different at $\mathrm{p}<0.05$. $\mathrm{L}=$ Light hours, $\mathrm{D}=$ Dark hours, $\mathrm{C}=$ Continuous, $\mathrm{I}=$ Intermittent, $\mathrm{GH}=$ Growth hormone, T3=Tri-iodothyronine, $\mathrm{T} 4=$ Tetra-iodothyronine, ND=Newcastle, $\mathrm{SE}=$ Standard error

Table-6: Logarithmic bacterial counts (total bacterial and Enterobacteriaceae counts Mean $\pm \mathrm{SE}$ ) in broilers exposed to lighting regimens using different lighting colors and durations.

\begin{tabular}{|c|c|c|c|}
\hline Light color & Photo-period L: D hour & Log. TBC CFU/mL & Log. TEC CFU/mL \\
\hline Red & & $5.25^{\mathrm{a}} \pm 0.022$ & $2.22^{a} \pm 0.022$ \\
\hline Blue & & $3.81^{c} \pm 0.013$ & $0.85^{c} \pm 0.044$ \\
\hline White & & $4.37^{b} \pm 0.011$ & $1.31^{b} \pm 0.025$ \\
\hline $\mathrm{p}$-value & & 0.000 & 0.000 \\
\hline \multicolumn{4}{|c|}{ Light's color $X$ lighting program } \\
\hline \multicolumn{4}{|l|}{ Red } \\
\hline & $C-23: 1$ & $5.23^{c} \pm 0.041$ & $2.06^{c} \pm 0.029$ \\
\hline & $C-18: 6$ & $5.25^{\mathrm{b}} \pm 0.038$ & $2.18^{\mathrm{b}} \pm 0.021$ \\
\hline & $\mathrm{I}-16: 8$ & $5.26^{a} \pm 0.036$ & $2.43^{\mathrm{a}} \pm 0.012$ \\
\hline \multicolumn{4}{|l|}{ Blue } \\
\hline & $C-23: 1$ & $3.71^{c} \pm 0.015$ & $0.51^{c} \pm 0.064$ \\
\hline & $C-18: 6$ & $3.80^{\mathrm{b}} \pm 0.012$ & $0.79^{\mathrm{b}} \pm 0.038$ \\
\hline & $\mathrm{I}-16: 8$ & $3.94^{\mathrm{a}} \pm 0.009$ & $1.27^{\mathrm{a}} \pm 0.011$ \\
\hline \multicolumn{4}{|l|}{ White } \\
\hline & $C-23: 1$ & $4.30^{c} \pm 0.019$ & $1.20^{\wedge} \pm 0.049$ \\
\hline & C- $18: 6$ & $4.36^{b} \pm 0.016$ & $1.31^{\mathrm{b}} \pm 0.038$ \\
\hline & $\mathrm{I}-16: 8$ & $4.44^{a} \pm 0.014$ & $1.43^{\mathrm{a}} \pm 0.029$ \\
\hline $\mathrm{p}$-value & & 0.004 & 0.000 \\
\hline
\end{tabular}

Means carrying different superscripts in the same column are significantly different at $p \leq 0.05$ or highly significantly different at $p<0.01$. Means carrying the same superscripts in the same column are non-significantly different at $p<0.05$. $\mathrm{L}=$ Light hours, $\mathrm{D}=$ Dark hours, $\mathrm{C}=$ Continuous, $\mathrm{I}=$ Intermittent, TBC=Total bacterial count, TEC=Total Enterobacteriaceae count, SE=Standard error 
the grow out. Light sources have been modified over the years from incandescent and fluorescent lamps to LED for their improving influence over growth performance as recorded by Kim et al. [26] and Riber [27].

In our study, red and blue LED lights were evaluated against standard medium white LED light. The results have shown that the blue monochromatic LED light enhanced production performance and CW compared to white and red lighting systems. These improvements might be attributed to the calming influence of blue light, and thus directed energy toward WG and FCR compared to red and white lights. Many researchers suggested that using blue light (single or combined) has numerous advantages as Abdel-Azeem and Borham, [28] who agreed with our results while testing the influence of LED red, blue, green, white, and mixed lights and found that blue LED lights with bird density $10 / \mathrm{m}^{2}$ were able to keep broilers calmers with synergistic influence on productive performance. Zhang et al. [29] declared that monochromatic blue LED light improved LBW and pectoral muscle growth in broilers. In addition, Archer [30] found that blue LED lights emitted cool temperature and were able to improve WG and food conversion ratio, as well as mitigate the impact of stresses and fear. On the contrary, Son and Ravindran [31] and Assaf et al. [32] recorded no significant influence on the WG of broilers supplied with three different colors of light (white, blue, or red).

Abdo et al. [33] recommended using monochromatic blue light in the summertime for its significant role in modifying heat shock biomarker activities toward enhancing immunity levels and reduce the negative impacts of heat stress. Cao et al. [34] also agreed and found a higher growth rate and carcass quality in broilers exposed to blue or green rather than red and white.

The current results revealed a significant improvement in GH, T3, T4, and insulin serum levels in broilers reared in blue compared to white and red LED lights. T3, T4, and insulin usually affect homeostasis; they increased in response to increased GLUCO, TG, and TC in blood. Since broiler activity was limited in blue than in red and white lights, GLUCO, TG, and TC levels were higher, and so the metabolic hormonal levels increased to increase metabolic activity and direct energy toward muscular development. Physiologically, GH is secreted in response to photoperiod with lower body activity, conditions that were available in broilers reared in blue light. The results were supported by those of Zhang et al. [35] who studied the influence of white, red, green, and blue LED lights on broiler's growth. They found GHRH proteins in hypothalamus and plasma GH levels increased in broilers reared in green and blue lights by $6.83-31.36 \%$. Yu et al. [36] recorded that T4 and testosterone hormones increased significantly in broilers exposed to the appropriate intensity of green and blue lights. On the contrary, Kumar [37] disagreed with our findings, reporting no significant differences in GLUCO, total protein, blood urea nitrogen, glutathione peroxidase, and superoxide dismutase among broilers exposed to blue, green, and white lights.

Blue LED light in our study improved the titer of anti-ND vaccine antibodies with significant reduction of intestinal bacterial load. The results were supported with those of Li et al. [38] who revealed the ability of green and blue lights to improve blood antioxidant (total antioxidant capacity, superoxide dismutase, and glutathione peroxidase) and subsequently increase B-lymphocyte proliferation in bursa of Fabricius in broilers depending on enhanced melatonin levels. Zhang et al. [39] agreed with our results and recorded a significant increase in anti-ND antibodies and elevated proliferation of $\mathrm{B}$ and T-lymphocytes in broilers exposed to blue monochromatic light by $11.9-40.3 \%$ and $10.4-36.2 \%$, respectively. They also recommended a combination of green and blue LED light to enhance the immune function of broilers. Chen et al. [40] reported that blue and green lights can induce T-lymphocyte proliferation by $9.57 \%$ $32.03 \%$ in vivo and $34.30 \%-50.53 \%$ in vitro trial. Guo et al. [41] agreed with our findings; they found an improvement of a-Naphthyl-acetate esterase and increased antibody production in broilers exposed to intermediate or low-intensity blue lights for the increased melatonin or activation of $\mathrm{T}$ and $\mathrm{B}$ lymphocyte proliferation.

Circadian rhythm control by the pineal gland is usually affected by the light to dark durations. Our study investigated three lighting regimens; continuous 23L:1D, continuous 18L:6D, and intermittent 16L:8D (4L:2D, 4 times). The results revealed a significant improvement in performance parameters, growth and metabolic hormones, and biochemical and immune profile in broilers reared in continuous $23 \mathrm{~L}: 1 \mathrm{D}$, and continuous 18L:6D compared to intermittent 16L:8D. Honda et al. [42] tested continuous $12 \mathrm{~h}$ white and $12 \mathrm{~h}$ blue light and found no disruption in the circadian rhythm of broilers. Lilburn and Loeffler [43] also agreed and stated that continuous lighting regimen is required for the development of digestive organs and WG in broilers. Dereli et al. [44] agreed with our findings; they tested 23L:1D or increasing duration of light and light intensity 20 lux (reduced intensity) in 272 male Ross broilers, they found enhanced performance parameters and carcass quality characteristics in broilers exposed to increased photoperiod with reasonable intensity. They also found enhanced resistant against environmental stressors.

Sun et al. [45] disagreed with our results, they evaluated 16L:8D, 23L:1D, decreasing-increasing (Dec-Inc), and intermittent 3L:1D and found lower tendency in Dec-Inc regimen for abdominal fat deposition, larger testis, comb percentage, and higher testosterone levels compared to intermittent 3L:1D and 23L:1D regimens. Furthermore, Olanrewaju et al. [46] did not agree with our results; 
they evaluated three photoperiods long-continuous 23L:1D, regular-intermittent 2L:2D, and short-non-intermittent $8 \mathrm{~L}: 16 \mathrm{D}$ from day 8 to day 56 . They found a significant increase $(\mathrm{p} \leq 0.05)$ in $\mathrm{LBW}, \mathrm{BWG}, \mathrm{FI}$, and $\mathrm{CW}$ in broiler reared in short-non-intermittent 8L:16D regimen, suggesting that regular-intermittent $2 \mathrm{~L}: 2 \mathrm{D}$ regimen can replace long-continuous $23 \mathrm{~L}: 1 \mathrm{D}$ one in broiler houses to save energy.

\section{Conclusion}

Blue LED light was able to improve significantly productive performance, $\mathrm{CW}$, some biochemical parameters, growth and metabolic hormones, and efficiency of Newcastle vaccine, as well as significantly reduce intestinal bacterial load compared to traditional white and red LED lights. Continuous 18L: 6D hours and 23L: 1D hours regimens at medium accepted intensity prove their efficacy in delivering the influence of blue light on the measured parameters, as well as enhanced growth and metabolism through their direct influence on hypothalamic hormones.

\section{Authors' Contributions}

ESS designed the experiment, participated, supervised the execution, and participated in writing the manuscript. RAH participated in the execution of the experiment, and in writing the manuscript. All authors read and approved the final manuscript.

\section{Acknowledgments}

A speechless thanking should be provided to Prof. MA Sobieh for his thankful directions during the execution of the work. A second thanking should be directed to laboratory technicians of Ismailia University hospital for their help in assessing sera samples for immunological examination. The authors did not receive any fund for this study.

\section{Competing Interests} interests.

The authors declare that they have no competing

\section{Publisher's Note}

Veterinary World remains neutral with regard to jurisdictional claims in published institutional affiliation.

\section{References}

1. Egbuniwe, I.C. and Ayo, J.O. (2016) Physiological roles of avian eyes in light perception and their responses to photoperiodicity. Worlds Poult. Sci. J., 72(3): 605-614.

2. Bovera, F., Lestingi, A., Piccolo, G., Iannaccone, F., Attia, Y.A. and Tateo, A. (2013) Effect of water restriction on growth performance, feed nutrient digestibility, carcass and meat traits of rabbits. Animal, 7(10): 1600-1606.

3. Olanrewaju, H.A., Collier, S.D., Purswell, J.L. and Branton, S.L. (2016) Effects of light sources and intensity on broilers grown to heavy weights: Hematophysiological and biochemical assessment. Int. J. Poult. Sci., 15(10): 384-393.

4. Jácome, I., Rossi, L.A. and Borille, R. (2014) Influence of artificial lighting on the performance and egg quality of commercial layers: A review. Rev. Bras. Cienc. Avic., 16(4): 337-344.
5. Olanrewaju, H.A., Purswell, J.L., Maslin, W.R., Collier, S.D. and Branton, S.L. (2015) Effects of color temperatures (kelvin) of LED bulbs on growth performance, carcass characteristics and ocular development indices of broilers grown to heavy weights. Poult. Sci., 94(3): 338-344.

6. El-Sabrout, K. and Khalil, M.H. (2017) Effect of LED lighting on hatchability and chick performance of chicken eggs. Pak. J. Zool., 49(6): 2323-2325.

7. Cao, J., Liu, W., Wang, Z., Xie, D., Jia, L. and Chen, Y. (2008) Green and blue monochromatic lights promote growth and development of broilers via stimulating testosterone secretion and myofiber growth. Poult. Sci., 17(2): 211-218.

8. Mohamed, R.A., Eltholth, M.M. and El-Saidy, N.R. (2014) Rearing broiler chickens under monochromatic blue light improve performance and reduce fear and stress during pre-slaughter handling and transportation. Biotechnol. Anim. Husbandry, 30(3): 457-471.

9. Yang, Y., Yu, Y., Pan, J., Ying, Y. and Zhou, H. (2016) A new method to manipulate broiler chicken growth and metabolism: Response to mixed LED light system. Sci. Rep., 6(1): 25972.

10. Olanrewaju, H.A., Purswell, J.L., Collier, S.D. and Branton, S.L. (2013) Interactive effects of photoperiod and light intensity on blood physiological and biochemical reactions of broilers grown to heavy weights. Poult. Sci., 92(4): 1029-1039.

11. Skrbic, Z., Pavlovski, Z., Lukic, M. and Petricevic, V. (2015) Incidence of footpad dermatitis and hock burns in broilers as affected by genotype, lighting program and litter type. Ann. Anim. Sci., 15(2): 433-445.

12. Karman, M. and Öcal, M. (2018) The effect of light regimen and feeding time on growth performance and mortality rate in broilers. Turk. J. Agric. Food Sci. Technol., 6(1): 91-96.

13. Olanrewaju, H.A., Miller, W.W., Maslin, W.R., Collier, S.D., Purswell, J.L. and Branton, S.L. (2016) Effects of light sources and intensity on broilers grown to heavy weights. Part 1: Growth performance, carcass characteristics and welfare indices. Poult. Sci., 95(4): 727-735.

14. Soliman, E.S., Sallam, N.H. and Abouelhassan, E.M. (2018) Effectiveness of poultry litter amendments on bacterial survival and Eimeria oocyst sporulation. Vet. World, 11(8): 1064-1073.

15. National Research Council. (1994) Nutrient Requirements for Poultry. $9^{\text {th }}$ ed. National Research Council, New York, USA.

16. Thrusfield, M. (2007) Sampling in Veterinary Epidemiology. $3^{\text {rd }}$ ed. Blackwell Science Ltd., London. p214-256.

17. Soliman, E.S. and Hassan, R.A. (2017) Evaluation of superphosphate and meta-bisulfide efficiency in litter treatment on productive performance and immunity of broilers exposed to ammonia stress. Adv. Anim. Vet. Sci., 5(6): 253-259.

18. Soliman, E.S., Hamad, R.T. and Ahmed, A. (2017) Prophylactic and immune modulatory influences of Nigella sativa Linn. In broilers exposed to biological challenge. Vet. World, 10(12): 1447-1455.

19. Wu, Y.N., Yan, F.F., Hu, J.Y., Chen, H., Tucker, C.M., Green, A.R. and Cheng, H.W. (2017) The effect of chronic ammonia exposure on acute-phase proteins, immunoglobulin, and cytokines in laying hens. Poult. Sci., 96(6): 1524-1530.

20. American Public Health Association, American Water Works Association, and Water Environment Federation. (2012) Standard Methods for the Examination of Water and Wastewater. $22^{\text {th }}$ ed. American Water Work Association Publications, Washington, DC.

21. Kim, S.K. and Lee, J.H. (2016) Biofilm modeling systems. Korean J. Microbiol., 52(2): 125-139.

22. Soliman, E.S., Moawed, S.A. and Ziaan, A.M.G. (2016) Assessing cleaning and disinfection regime in a slaughterhouse against carcasses contamination. Adv. Anim. Vet. Sci., 4(9): 449-457. 
23. Murray, P.R., Rosenthal, K.S. and Pfaller, M.A. (2015) Medical Microbiology. $8^{\text {th }}$ ed. Elsevier Health Sciences, Philadelphia, PA, USA.

24. Lorenzo-Sava, U. and Ferrando, P.J. (2015) POLYMAT-C: A comprehensive SPSS program for computing the polychoric correlation matrix. Behav. Res. Methods, 47(3): 884-889.

25. Olanrewaju, H.A., Purswell, J.L., Collier, S.D. and Branton, S.L. (2014) Effects of genetic strain and light intensity on blood physiological variables of broilers grown to heavy weights. Poult. Sci., 93(4): 970-978.

26. Kim, N., Lee, S.R. and Lee, S.J. (2014) Effects of light color on energy expenditure and behavior in broiler chickens. Asian Australas. J. Anim. Sci., 27(7): 1044-1049.

27. Riber, A.B. (2015) Effects of color of light on preferences, performance, and welfare in broilers. Poult. Sci., 94(8): $1767-1775$.

28. Abdel-Azeem, A.F. and Borham, B.E. (2018) Productive and physiological response of broiler chickens exposed to different colored light-emitting diode and reared under different stocking densities. Egypt. Poult. Sci. J., 38(4): 1243-1264.

29. Zhang, L., Zhang, H.J., Qiao, X., Yue, H.Y., Wu, S.G., Yao, J.H. and Qi, G.H. (2012) Effect of monochromatic light stimuli during embryogenesis on muscular growth, chemical composition, and meat quality of breast muscle in male broilers. Poult. Sci., 91(4): 1026-1031.

30. Archer, G.S. (2018) Color temperature of light-emitting diode lighting matters for optimum growth and welfare of broiler chickens. Animals, 12(5): 1015-1021.

31. Son, J.H. and Ravindran, V. (2009) Effect of Light Colour on the Behavior and Performance of Broilers. Poultry Welfare Symposium, Cervia, Italy.

32. Assaf, W., Mohra, I. and Hashem, Y. (2015) Effect of light color on some of performance indices of hybrid cup 500-broilers. Int. J. Poult. Sci., 14(2): 100-102.

33. Abdo, S.E., El-Kassas, S., El-Nahas, A.F. and Mahmoud, S. (2017) Modulatory effect of monochromatic blue light on heat stress response in commercial broilers. Oxid. Med. Cell. Longev., 2017(1): 1-13.

34. Cao, J., Wang, Z., Dong, Y., Li, J., Li, F. and Chen, Y. (2012) Effect of combinations of monochromatic lights on growth and productive performance of broilers. Poult. Sci., 91(12): 3013-3018.

35. Zhang, L., Cao, J., Wang, Z., Dong, Y. and Chen, Y. (2016) Melatonin modulates monochromatic light-induced GHRH expression in the hypothalamus and GH secretion in chicks. Acta Histochem., 118(3): 286-292.

36. Yu, Y., Li, Z., Zhong, Z., Jin, S., Pan, J., Rao, X. and Yu, Y. (2018) Effect of monochromatic green LED light stimuli during incubation on embryo growth, hatching performance, and hormone levels. Am. Soc. Agric. Biol. Eng., 61(2): 661-669.

37. Kumar, S. (2016). Evaluation of Broilers Performance under Coloured Light Emitting Diodes. Master Thesis, Department of Livestock Production Management, College of Veterinary Medicine. Ch. 2. Guru Angad Dev Veterinary and Animal Sciences University, Ludhiana, India. p2-4.

38. Li, J., Cao, J., Wang, Z., Dong, Y. and Chen, Y. (2015) Melatonin plays a critical role in inducing B lymphocyte proliferation of the bursa of Fabricius in broilers via monochromatic lights. J. Photochem. Photobiol. B, 142(1): 29-34.

39. Zhang, Z., Cao, J., Wang, Z., Dong, Y. and Chen, Y. (2014) Effect of a combination of green and blue monochromatic light on broiler immune response. J. Photochem. Photobiol. B., 138(9): 118-123.

40. Chen, F., Reheman, A., Cao, J., Wang, Z., Dong, Y., Zhang, Y. and Chen, Y. (2016) Effect of melatonin on monochromatic light-induced T-lymphocyte proliferation in the thymus of chickens. J. Photochem. Photobiol. B., 161(8): 9-16

41. Guo, Y.L., Ma, S.M., Du, J.J. and Chen, J.L. (2018) Effects of light intensity on growth, anti-stress ability and immune function in yellow feathered broilers. Rev. Bras. Cienc. Avic., 20(1): 79-84.

42. Honda, K., Kondo, M., Hiramoto, D., Saneyasu, T. and Kamisoyama, H. (2017) Effects of continuous white light and $12 \mathrm{~h}$ white- $12 \mathrm{~h}$ blue light-cycles on the expression of clock genes in diencephalon, liver, and skeletal muscle in chicks. Comp. Biochem. Physiol. A Mol. Integr. Physiol., 207(1): 73-78

43. Lilburn, M.S. and Loeffler, S. (2015) Early intestinal growth and development in poultry. Poult. Sci., 94(7): 1569-1576.

44. Dereli, F.E., Nazligül, A., Türkyilmaz, M.K., Karaarslan, S. and Kaya, M. (2017) Effects of photoperiod length and light intensity on performance, carcass characteristics and heterophil to lymphocyte ratio in broilers. Kafkas Univ. Vet. Fak. Derg., 23(1): 39-45.

45. Sun, Y.Y., Tang, S., Chen, Y., Li, D.L., Bi, Y.L., Hua, D.K., Chen, C., Luo, Q.Y., Yang, L. and Chen, J.L. (2017) Effects of light regimen and nutrient density on growth performance, carcass traits, meat quality, and health of slow-growing broiler chickens. Livest. Sci., 198(4): 201-208.

46. Olanrewaju, H.A., Miller, W.W., Maslin, W.R., Collier, S.D., Purswell, J.L. and Branton, S.L. (2018) Influence of light sources and photoperiod on growth performance, carcass characteristics, and health indices of broilers grown to heavy weights. Poult. Sci., 97(4): 1109-1116. 